\title{
Intervention for prehypertension and its cardiovascular risk factors in Inner Mongolia
}

\author{
X.S. Zhao, R. Wang, L.R. Bin and S.Q.G. Wa \\ Department of Cardiology, Inner Mongolia Hospital, \\ Inner Mongolia Medical College, Hohhot, China \\ Corresponding author: X.S. Zhao \\ E-mail: xingshengzhao@126.com \\ Genet. Mol. Res. 13 (3): 4867-4882 (2014) \\ Received February 13, 2013 \\ Accepted November 1, 2013 \\ Published July 4, 2014 \\ DOI http://dx.doi.org/10.4238/2014.July.4.1
}

\begin{abstract}
Blood pressure levels were evaluated among prehypertension patients with associated cardiovascular risk factors to determine the effect of different interventions with respect to new endpoint events. A total of 1112 patients were equally and randomly divided into control, lifestyle, drug, and lifestyle + drug groups, and were followed-up for 12 months. We collected the age, height, weight, blood pressure, heart rate, blood lipid levels, blood glucose levels, and other clinical data from all subjects at the baseline and at the end of the follow-up period. The average systolic blood pressure (SBP) and diastolic blood pressure (DBP) in the intervention groups were significantly reduced after the intervention (lifestyle + drug $>$ drug $>$ lifestyle). SBP and DBP were higher in the control group than before intervention $(\mathrm{P}<0.05)$. Age, gender, heart rate, triglyceride (TG) levels, body mass index (BMI), alcohol consumption, and family history were the major factors affecting blood pressure. Increased BMI, TG, high-sensitivity C-reactive protein, alcohol consumption, family history, SBP, and DBP were major risk factors of hypertension. At the end of the follow-up period, there were 67 cases of hypertension, 32 cases of diabetes, and 12 cases of the endpoint events. Hypertension and diabetes events were reduced
\end{abstract}


in the intervention groups relative to the control group $(\mathrm{P}<0.05)$. The number of cases with incident cardiovascular endpoints did not differ among the three intervention groups $(\mathrm{P}>0.05)$. Therefore, hypertension and related cardiovascular events can be controlled with different interventions.

Key words: Normal high blood pressure; Risk factors; Intervention studies

\section{INTRODUCTION}

In recent years, prehypertension $(\mathrm{PH})$ was introduced into the classification of blood pressure (BP), referring to BPs of 120/80-139/89 mmHg. Patients with PH have double the risk of developing hypertension in the future compared to individuals with lower BP (Chobanian et al., 2003). The PH population is a high-risk group who are likely to develop hypertension, cardiovascular, and cerebrovascular diseases. The risk of cardiovascular and cerebrovascular diseases is increased if $\mathrm{PH}$ is accompanied by high cholesterol, high blood sugar, obesity, or one or more cardiovascular risk factors. Epidemiological studies have shown that in the 35- to 64-year-old population, the occurrence rate of $\mathrm{PH}$ is $32.2 \%$ (Sun and Zhao, 2005). In the middle-aged population, the incidence of hypertension for BPs between 120-129/80-84 mmHg and 130-139/85-89 $\mathrm{mmHg}$ are 45 and 64\%, respectively (Zhao et al., 2006). In an 11-year follow-up study of 7000 normotensive individuals, Vasan et al. (2001) found an incidence of $25 \% \mathrm{PH}$, which showed 1.5- to 2.5-fold increased chance of stroke or heart failure compared to the normal population.

Currently, lifestyle interventions for the $\mathrm{PH}$ population, such as salt restriction, increased exercise, and smoking cessation, can be effective in reducing BP levels; however, certain difficulties remain for the general implementation and long-standing aspects. Drug intervention can delay the incidence of hypertension. The TROPHY trial in the United States showed that angiotensin receptor blocker therapy could reduce the incidence of hypertension in the PH population (Julius et al., 2006). It was postulated that due to the increased cardiovascular risk in the $\mathrm{PH}$ population, antihypertensive therapy and early intervention can effectively control these risk factors and prevent the development of hypertension, which was also important for the primary prevention of strokes and coronary heart diseases (Wang and Wang, 2004; Chockalingam et al., 2005; Tsai et al., 2005; Choi et al., 2006; Grotto et al., 2006).

Inner Mongolia Autonomous Region is a hypertension-prone area, and the incidence of PH is high. The expected effect of early intervention of $\mathrm{PH}$ patients will be far greater than for those who have already developed hypertension with multiple risk factors. This study was designed to conduct 12-month follow-up observations in residents of more than 3 years, aged 20-59, without cardiovascular diseases, but with PH. Residents were recruited from urban, suburban, rural, semi-agricultural, and pastoral areas of five randomly chosen cities in the Inner Mongolia Autonomous Region. The PH population was divided into the control group and three intervention groups: lifestyle, drugs, and lifestyle + drugs. BP levels and biochemical changes were compared under different modes of intervention, and data were analyzed with statistical methods (Liu et al., 2010; Fang et al., 2010; Sun et al., 2007, 2010; Wang et al., 2011). 


\section{MATERIAL AND METHODS}

\section{Patients}

Inclusion criteria of patients were based on epidemiological investigations conducted from 2009 to 2010 at the Inner Mongolia Autonomous Region Hospital, Baotou Central Hospital, Ordos Central Hospital, Chifeng City Center hospital, and the Zhengxiang Baiqi Hospital, in line with diagnostic criteria of PH associated with cardiovascular risk factors. Written informed consent was obtained from all participants.

The exclusion criteria included the following: previous diagnosis of hypertension or taking antihypertensive drugs; previous infarction, cerebral hemorrhage, subarachnoid hemorrhage; previous myocardial infarction; obvious liver and kidney dysfunction, acute inflammatory diseases, cancer, or acute vascular events within the past 3 months; PH was not associated with cardiovascular risk factors. The following conditions were observed during follow-up: cerebral infarction, cerebral hemorrhage, subarachnoid hemorrhage; myocardial infarction; death from any cause; transferred out, retired, or moved to the field; refused to participate in the experiment.

Patients that did not record BP readings after taking medication or who did not take the drugs prescribed were also excluded.

\section{Experimental design}

This study was designed based on selected survey questionnaires and physical examinations. Information was collected by trained medical personnel at the same place for the same population, according to the order of the first inspection for the second follow-up examination, survey content, and anthropometric biochemical parameters. Subjects who received lifestyle intervention were asked to carefully fill in their specific implementation measures, and those who received drug intervention were required to fill out detailed medication reports. The period of follow-up was 12 months, at which point the data were assessed for quality, and statistical analysis was performed. During the experimental design phase, the survey followup stage, the stage of data entry and analysis, instrument calibration and ethical issues were under strict quality control.

The survey mainly included information related to the general living condition of the family, basic individual condition, basic state of health, behavior, health awareness, and family history. Physical examinations included waist and height measurements in order to determine body mass and body mass index (BMI). Blood tests were conducted to determine fasting plasma glucose (FPG), total cholesterol (TC), triglycerides (TG), high-density lipoprotein cholesterol (HDL-C), and low-density lipoprotein cholesterol (LDL-C), which were measured using an automatic biochemical analyzer (Abbott Laboratories). The C-reactive protein (CRP) content was detected by enzyme-linked immunosorbent assay (ELISA). Specific experimental procedures were performed in accordance with manufacturer instructions.

\section{Diagnostic criteria}

The PH value was based on diagnostic criteria established in 2003 (Prevention, De- 
tection, Evaluation and Treatment of Hypertension, the National Committee Seventh Report (JNC-7): systolic blood pressure (SBP) between 120 and $139 \mathrm{mmHg}(1 \mathrm{mmHg}=0.133 \mathrm{kPa})$ and/or diastolic blood pressure (DBP) between 80 and $89 \mathrm{mmHg}$. Hypertension was based on diagnostic criteria of the 2005 hypertension guidelines: SBP $\geq 140 \mathrm{mmHg}$ and/or $\mathrm{DBP} \geq 90$ $\mathrm{mmHg}$, or if a subject was taking antihypertensive drugs.

\section{Grouping and intervention}

A total of 1112 individuals with PH were randomly divided into four groups: the lifestyle group, drug group, lifestyle + drug group, and control group, with 278 cases in each group. The drug intervention involved Compound Hypotensive Tablets (Beijing Double-Crane Pharmaceutical Co., Ltd.) under a usual dose of one tablet per day at 8:00 am by oral administration and a maintenance dose of one tablet per day for 2 to 3 days. During the study period, researchers gradually adjusted the dosage according to the subjects' tolerance. Every patient filled out a questionnaire related to adverse effects. Patients who could not tolerate the medication withdrew from the study. The Compound Hypotensive Tablets are the specified medication of the National Eleventh Five-Year Technology Support Program of the "China Prehypertension with Cardiovascular Risk Factors Intervention" in "Hypertension Prevention". The lifestyle intervention included smoking cessation, abstinence, salt restriction, proper diet, and sports perseverance to control obesity. Moreover, lectures, leaflet distribution, and lifestyle guidance were provided to inform patients on the potential outcomes and hazards of $\mathrm{PH}$.

\section{Observation indices}

Demographic information included age, gender, race, BP, heart rate, height, weight, BMI, smoking, alcohol consumption, diet, and exercise. The following biochemical indices were calculated: FPG, TC, HDL-C, LDL-C, TG, and high-sensitivity (hs)-CRP. The primary endpoint was a BP $\geq 140 / 90 \mathrm{mmHg}$, and the secondary endpoint was stroke. Total cardiovascular events were also recorded, which included cerebral infarction, cerebral hemorrhage, angina pectoris, acute myocardial infarction, and sudden cardiac death. The third-level endpoints included all causes of mortality, diabetes, and new onset of cancer. Diet compliance and exercise compliance adopted the four-level evaluation method. Compliance in the drug group was determined using the Morisky questionnaire. The safety of the medication was determined according to the provisions of Article 29 in the "Adverse Drug Reaction Reporting" and "Monitoring of the Management Approach".

\section{Statistical methods}

The SPSS 13.0 analysis software was used for statistical analyses. Data are reported as means \pm standard deviation. The data between two groups were compared with analysis of variance (ANOVA). Partial correlation analysis was adopted to determine the relationships between two variables. The effects of potentially influencing factors were controlled through multiple linear regressions. Logistic stepwise regression analysis was used for multivariate analyses. $\mathrm{P}<0.05$ was considered to be statistically significant. 


\section{RESULTS}

\section{Baseline information of subjects and indicator data}

The baseline time point was from 2009 to 2010 . A total of 1112 cases aged 20-59 years (mean $43 \pm 9$ years) with PH were screened. Five hundred and forty-three patients were males $(48.8 \%)$ and 569 patients were females $(51.2 \%)$. A total of 358 patients were between 30 and 39 years old, and 349 patients ranged from 0 to 49 years old. The remaining 62 patients were between 20 and 29 years old.

\section{General data analysis of subjects before intervention}

Prior to intervention, there were no significant differences between groups $(\mathrm{P}>0.05)$ in gender, age, SBP, DBP, heart rate, height, weight, BMI, waist circumference (WC), TG, TC, LDLC, FPG, hs-CRP, smoking, alcohol consumption, history of diabetes, or family history, based on both the F-test of ANOVA and paired comparisons with Bonferroni's correction (Table 1).

Table 1. General data analysis before intervention.

\begin{tabular}{|c|c|c|c|c|c|c|}
\hline Stems & Control & Lifestyle & Drug & Lifestyle + drug & $\mathrm{F}$ & $\mathrm{P}$ \\
\hline Gender & $1.45 \pm 0.50$ & $1.54 \pm 0.50$ & $1.55 \pm 0.50$ & $1.51 \pm 0.50$ & 2.18 & 0.89 \\
\hline Age (years) & $43.94 \pm 9.52$ & $43.49 \pm 9.35$ & $43.82 \pm 9.03$ & $43.53 \pm 9.81$ & 0.15 & 0.93 \\
\hline Heart rate (bpm) & $74.74 \pm 9.26$ & $74.85 \pm 8.62$ & $74.60 \pm 8.02$ & $74.31 \pm 8.53$ & 0.21 & 0.89 \\
\hline Height (m) & $1.67 \pm 0.08$ & $1.66 \pm 0.08$ & $1.66 \pm 0.07$ & $1.66 \pm 0.08$ & 1.97 & 0.12 \\
\hline Weight (kg) & $68.9 \pm 10.83$ & $68.4 \pm 10.88$ & $68.1 \pm 10.21$ & $68.4 \pm 11.21$ & 0.26 & 0.85 \\
\hline BMI $\left(\mathrm{kg} / \mathrm{m}^{2}\right)$ & $24.69 \pm 3.07$ & $24.67 \pm 3.07$ & $24.79 \pm 2.70$ & $24.72 \pm 3.29$ & 0.08 & 0.97 \\
\hline $\mathrm{WC}(\mathrm{cm})$ & $83.83 \pm 7.95$ & $84.11 \pm 7.55$ & $83.94 \pm 7.80$ & $84.08 \pm 8.13$ & 0.08 & 0.97 \\
\hline $\mathrm{TG}(\mathrm{mg} / \mathrm{dL})$ & $1.70 \pm 1.02$ & $1.65 \pm 1.05$ & $1.66 \pm 1.04$ & $1.68 \pm 1.12$ & 0.15 & 0.93 \\
\hline $\mathrm{TC}(\mathrm{mg} / \mathrm{dL})$ & $4.87 \pm 0.95$ & $4.82 \pm 0.97$ & $4.90 \pm 0.92$ & $4.89 \pm 0.99$ & 0.46 & 0.71 \\
\hline LDL-C (mg/dL) & $2.46 \pm 0.85$ & $2.48 \pm 0.86$ & $2.55 \pm 0.93$ & $2.51 \pm 0.90$ & 0.56 & 0.65 \\
\hline $\mathrm{FPG}(\mathrm{mg} / \mathrm{dL})$ & $5.38 \pm 1.52$ & $5.26 \pm 1.40$ & $5.35 \pm 1.39$ & $5.35 \pm 1.55$ & 0.36 & 0.78 \\
\hline $\mathrm{hs}-\mathrm{CRP}(\mathrm{mg} / \mathrm{dL})$ & $0.15 \pm 0.14$ & $0.14 \pm 0.06$ & $0.14 \pm 0.07$ & $0.14 \pm 0.05$ & 0.43 & 0.73 \\
\hline Smoking & $1.19 \pm 0.39$ & $1.21 \pm 0.41$ & $1.17 \pm 0.38$ & $1.17 \pm 0.36$ & 0.61 & 0.61 \\
\hline Drinking & $1.16 \pm 0.37$ & $1.16 \pm 0.37$ & $1.16 \pm 0.36$ & $1.16 \pm 0.37$ & 0.03 & 1.00 \\
\hline Diabete & $1.01 \pm 0.12$ & $1.01 \pm 0.12$ & $1.01 \pm 0.12$ & $1.01 \pm 0.12$ & 0.00 & 1.00 \\
\hline Family history & $1.03 \pm 0.16$ & $1.04 \pm 0.20$ & $1.04 \pm 0.20$ & $1.06 \pm 0.23$ & 1.26 & 0.29 \\
\hline
\end{tabular}

Data are reported as means \pm SD for 278 individuals in each group. BMI = body mass index; $\mathrm{WC}=$ waist circumference; $\mathrm{TG}=$ triglycerides; $\mathrm{TC}=$ total cholesterol; LDL-C = low-density lipoprotein cholesterol; FPG = fasting plasma glucose; hs-CRP = high-sensitivity $\mathrm{C}$-reactive protein.

\section{General data analysis of subjects after intervention}

The general data and statistical comparisons among groups following intervention are shown in Table 2.

After intervention, heart rate, body weight, BMI, TC, and LDL-C were not significantly changed based on the F-test (P > 0.05). However, SBP, DBP, WC, TG, FPG, hs-CRP, smoking, alcohol consumption, and history of diabetes were all significantly different following intervention $(\mathrm{P}<0.05)$. Paired comparisons with Bonferroni's correction revealed that SBP changed significantly following intervention, and DBP differed significantly between the control group and the three intervention groups, whereas there was no significant difference between the drug group and the lifestyle + drug group. WC did not change significantly after intervention in any group. TG and FPG showed significant differences between the control group and the lifestyle and lifestyle + drug groups, and 
pairwise differences between the control and drug groups, lifestyle and drug groups, or lifestyle + drug and drug groups were not significant. Similarly, hs-CRP differed significantly between the control group and the lifestyle and lifestyle + drug groups, and also showed significant pairwise differences between the lifestyle and drug groups and the lifestyle + drug and drug groups. Smoking and alcohol consumption differed significantly between the control group and the lifestyle and lifestyle + drug groups. Among the intervention groups, smoking and alcohol consumption differed significantly between the lifestyle and drug groups and between the lifestyle + drug and drug groups, whereas there were no pairwise differences between the control and drug groups or the lifestyle and lifestyle + drug groups $(\mathrm{P}>0.05)$. The proportion of subjects with a history of diabetes differed significantly between the control group and the lifestyle and lifestyle + drug groups, but no pairwise differences were observed between the control and drug groups, the lifestyle and drug groups, the lifestyle and lifestyle + drug groups, or the drug and lifestyle + drug groups.

\begin{tabular}{|c|c|c|c|c|c|c|}
\hline Variance & Control & Lifestyle & Drug & Lifestyle + drug & $\mathrm{F}$ & $P$ \\
\hline HR & $74.8 \pm 8.63$ & $74.6 \pm 9.26$ & $74.31 \pm 8.53$ & $74.5 \pm 7.87$ & 0.20 & 0.90 \\
\hline Weight & $69.03 \pm 10.75$ & $67.58 \pm 10.34$ & $68.1 \pm 10.15$ & $67.58 \pm 10.69$ & 1.18 & 0.32 \\
\hline BMI & $24.71 \pm 3.04$ & $24.37 \pm 2.92$ & $24.78 \pm 2.67$ & $24.40 \pm 3.15$ & 1.40 & 0.24 \\
\hline WC & $83.89 \pm 7.87$ & $82.48 \pm 7.28$ & $83.9 \pm 7.733$ & $82.29 \pm 7.86$ & 3.76 & 0.01 \\
\hline TG & $1.75 \pm 1.00$ & $1.52 \pm 0.8 \mathrm{a} 5$ & $1.68 \pm 1.03$ & $1.53 \pm 0.93$ & 3.98 & 0.00 \\
\hline $\mathrm{TC}$ & $4.81 \pm 0.96$ & $4.87 \pm 0.96$ & $4.90 \pm 0.99$ & $4.90 \pm 0.92$ & 0.50 & 0.68 \\
\hline LDL-C & $2.46 \pm 0.85$ & $2.48 \pm 0.86$ & $2.55 \pm 0.93$ & $2.52 \pm 0.90$ & 0.67 & 0.57 \\
\hline FPG & $5.56 \pm 1.87$ & $5.11 \pm 1.22$ & $5.40 \pm 1.46$ & $5.11 \pm 1.09$ & 6.72 & 0.00 \\
\hline hs-CRP & $0.15 \pm 0.13$ & $0.10 \pm 0.08$ & $0.13 \pm 0.11$ & $0.10 \pm 0.05$ & 14.7 & 0.00 \\
\hline Smoking & $1.19 \pm 0.39$ & $1.01 \pm 0.09$ & $1.17 \pm 0.38$ & $1.01 \pm 0.09$ & 35.9 & 0.00 \\
\hline Drinking & $1.16 \pm 0.37$ & $1.01 \pm 0.09$ & $1.15 \pm 0.36$ & $1.00 \pm 0.00$ & 32.4 & 0.00 \\
\hline Diabete & $1.03 \pm 0.17$ & $1.00 \pm 0.08$ & $1.02 \pm 0.15$ & $1.00 \pm 0.05$ & 4.97 & 0.00 \\
\hline
\end{tabular}

Data are reported as means \pm SD for 278 individuals in each group. For units and abbreviations, see Table 1.

\section{Comparison before and after intervention}

\section{SBP and DBP before and after intervention}

The mean SBP and DBP decreased significantly in the lifestyle, drug, and lifestyle + drug groups after intervention (lifestyle + drug $>$ drug $>$ lifestyle). In the control group, the mean SBP and DBP were higher after intervention. Before intervention, SBP and DBP did not differ significantly among groups based on the F-test and the paired comparisons. After intervention, however, both SBP and DBP differed significantly between the control group and the intervention groups $(\mathrm{P}<0.05$ in all pairwise comparisons). There was no significant difference in DBP between the lifestyle and drug intervention groups (Table 3 and Figure 1A).

\section{Weight, BMI, and WC before and after intervention}

Before the intervention, WC did not show significant differences among groups. After intervention, WC changed significantly $(\mathrm{P}<0.05)$ in the intervention groups, with no significant differences among the three groups (Table 4). The mean values of weight, BMI, and WC were all lower in the intervention groups following intervention, whereas these values all increased in the control group after intervention. The lifestyle and lifestyle + drug interventions had obvious impacts on weight, BMI, and WC, suggesting that these are effective intervention strategies. 
Table 3. Blood pressure comparison of subjects before and after intervention.

\begin{tabular}{|c|c|c|c|c|c|c|}
\hline Variance & Control & Lifestyle & Medicine & Lifestyle + medicine & $\mathrm{F}$ & $P$ \\
\hline \multicolumn{7}{|l|}{ SBP } \\
\hline 0 Week & $124.31 \pm 8.24$ & $124.78 \pm 8.22$ & $124.47 \pm 8.52$ & $124.07 \pm 8.28$ & 0.36 & 0.79 \\
\hline 12 Months & $125.65 \pm 9.33^{* \bullet}$ & $122.3 \pm 8.65^{* \Delta}$ & $120.32 \pm 8.28^{* \Delta \bullet}$ & $116.93 \pm 8.10^{* \bullet}$ & 50.11 & 0.00 \\
\hline \multicolumn{7}{|l|}{ DBP } \\
\hline 0 Week & $81.81 \pm 4.99$ & $81.77 \pm 5.05$ & $81.55 \pm 4.87$ & $80.80 \pm 5.08$ & 2.45 & 0.06 \\
\hline 12 Months & $82.63 \pm 4.80^{* \star}$ & $78.49 \pm 5.05^{* \Delta \bullet}$ & $77.99 \pm 4.39^{* \Delta \bullet}$ & $74.49 \pm 4.22^{* \bullet}$ & 14.61 & 0.00 \\
\hline
\end{tabular}

Data are reported as means \pm SD for 278 individuals in each group. $* \mathrm{P}<0.05$ compared with the control group; ${ }^{\Delta} \mathrm{P}$

$<0.05$ compared with the lifestyle + drug group; ${ }^{\bullet} \mathrm{P}<0.05$ comparison before and after treatment.
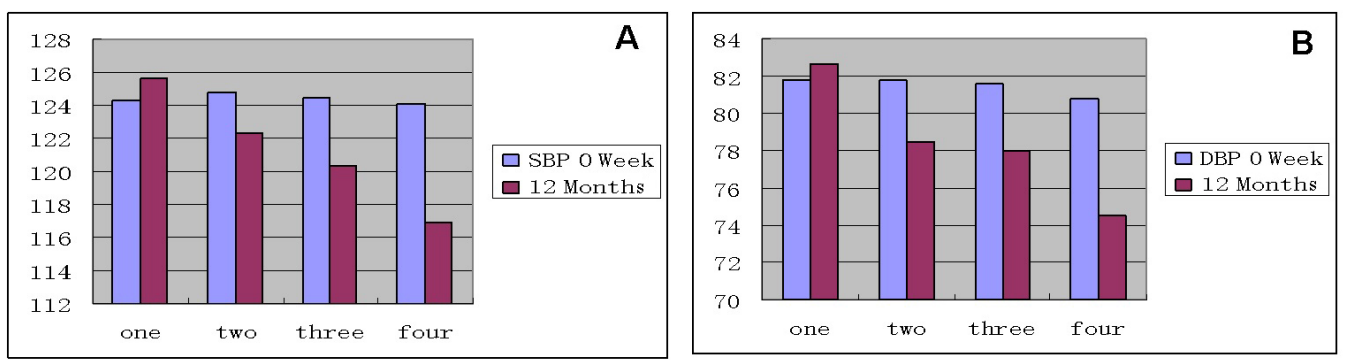

Figure 1. Mean values of SBP $(\mathbf{A})$ and DBP $(\mathbf{B})$ of the patients in each groups before and after intervention. One = non-intervention group; two = lifestyle group; three = drug group; four = lifestyle and drug group.

\begin{tabular}{|c|c|c|c|c|c|c|}
\hline Variance & Control & Lifestyle & Drug & Lifestyle + drug & $\mathrm{F}$ & $\mathrm{P}$ \\
\hline \multicolumn{7}{|l|}{ Weight } \\
\hline 0 Week & $68.9 \pm 10.83$ & $68.45 \pm 10.88$ & $68.17 \pm 10.21$ & $68.49 \pm 11.21$ & 0.26 & 0.65 \\
\hline 12 Months & $69.0 \pm 10.75$ & $67.58 \pm 10.34$ & $68.13 \pm 10.15$ & $67.58 \pm 10.69$ & 1.18 & 0.32 \\
\hline \multicolumn{7}{|l|}{ BMI } \\
\hline 0 Week & $24.69 \pm 3.07$ & $24.67 \pm 3.07$ & $24.79 \pm 2.70$ & $24.72 \pm 3.29$ & 0.08 & 0.97 \\
\hline 12 Months & $24.71 \pm 3.04$ & $24.37 \pm 2.92$ & $24.78 \pm 2.67$ & $24.40 \pm 3.15$ & 1.40 & 0.24 \\
\hline \multicolumn{7}{|l|}{ WC } \\
\hline 0 Week & $83.83 \pm 7.95$ & $84.11 \pm 7.55$ & $83.94 \pm 7.80$ & $84.08 \pm 8.13$ & 0.08 & 0.97 \\
\hline 12 Months & $83.89 \pm 7.87$ & $82.48 \pm 7.28$ & $83.96 \pm 7.73$ & $82.29 \pm 7.86$ & 3.76 & 0.11 \\
\hline \multicolumn{7}{|l|}{ TG } \\
\hline 0 Week & $1.70 \pm 0.06$ & $1.65 \pm 0.07$ & $1.66 \pm 0.08$ & $1.68 \pm 0.06$ & 0.15 & 0.93 \\
\hline 12 Months & $1.75 \pm 0.08$ & $1.52 \pm 0.06$ & $1.68 \pm 0.05$ & $1.53 \pm 0.04$ & 3.98 & 0.08 \\
\hline \multicolumn{7}{|l|}{$\mathrm{TC}$} \\
\hline 0 Week & $4.87 \pm 0.95$ & $4.82 \pm 0.97$ & $4.90 \pm 0.92$ & $4.90 \pm 0.99$ & 0.46 & 0.71 \\
\hline 12 Months & $4.81 \pm 0.96$ & $4.82 \pm 0.96$ & $4.90 \pm 0.99$ & $4.90 \pm 0.92$ & 0.50 & 0.68 \\
\hline \multicolumn{7}{|l|}{ LDL-C } \\
\hline 0 Week & $2.46 \pm 0.85$ & $2.48 \pm 0.87$ & $2.55 \pm 0.93$ & $2.51 \pm 0.90$ & 0.56 & 0.65 \\
\hline 12 Months & $2.46 \pm 0.86$ & $2.48 \pm 0.86$ & $2.55 \pm 0.93$ & $2.52 \pm 0.89$ & 0.67 & 0.57 \\
\hline \multicolumn{7}{|l|}{ FPG } \\
\hline 0 Week & $5.38 \pm 1.52$ & $5.26 \pm 1.40$ & $5.35 \pm 1.39$ & $5.35 \pm 1.55$ & 0.36 & 0.78 \\
\hline 12 Months & $5.56 \pm 1.87$ & $5.11 \pm 1.22$ & $5.40 \pm 1.46$ & $5.11 \pm 1.09$ & 6.72 & 0.00 \\
\hline \multicolumn{7}{|l|}{ hs-CRP } \\
\hline 0 Week & $0.15 \pm 0.04$ & $0.14 \pm 0.02$ & $0.14 \pm 0.05$ & $0.14 \pm 0.01$ & 0.43 & 0.73 \\
\hline 12 Months & $0.15 \pm 0.03$ & $0.10 \pm 0.01$ & $0.13 \pm 0.04$ & $0.10 \pm 0.04$ & 14.77 & 0.00 \\
\hline
\end{tabular}

Data are reported as means \pm SD for 278 individuals in each group. For units and abbreviations, see Table 1.

\section{Biochemical indicators before and after intervention}

Before intervention, TG, TC, LDL-C, FPG, and hs-CRP did not differ significantly 
among groups $(\mathrm{P}>0.05)$. After intervention, there were no significant differences in TC or LDL-C. However, TG, FPG, and hs-CRP differed significantly between the control group and the lifestyle and lifestyle + drug groups, but there was no difference between the control and drug intervention groups $(\mathrm{P}>0.05)$. Levels of hs-CRP differed significantly among all three intervention groups $(\mathrm{P}<0.05)$ (Table 4 and Figure $1 \mathrm{~B})$.

\section{Relationship between SBP and DBP in patients with PH and cardiovascular risk factors}

Partial correlation analysis was used to evaluate the effect of various indicators on SBP and DBP. SBP was significantly positively correlated with age, heart rate, TG, FPG, and family history $(\mathrm{r}=0.067,0.168,0.128,0.061$, and 0.104 , respectively, $\mathrm{P}<0.05)$. There was no association between SBP and gender, weight, height, WC, BMI, TC, HDL-C, LDL-C, FPG, hs-CRP, smoking, alcohol consumption, or history of diabetes $(\mathrm{P}>0.05)$ (Table 5).

\begin{tabular}{|c|c|c|c|c|c|c|c|c|}
\hline & Age & Weight & Height & WC & HR & BMI & TG & $\mathrm{TC}$ \\
\hline & 0.067 & 0.015 & 0.028 & 0.016 & 0.168 & 0.042 & 0.128 & 0.049 \\
\hline \multirow{2}{*}{ Sig. (2-tailed) } & 0.027 & 0.621 & 0.361 & 0.607 & 0.000 & 0.166 & 0.000 & 0.104 \\
\hline & HDL-C & LDL-C & FPG & hs-CRP & Smoking & Drinking & & \\
\hline $\mathrm{r}$ & 0.004 & 0.051 & 0.061 & 0.050 & 0.040 & 0.003 & & \\
\hline Sig. (2-tailed) & 0.882 & 0.094 & 0.044 & 0.100 & 0.186 & 0.919 & & \\
\hline
\end{tabular}

For abbreviations, see Table 1.

DBP was significantly positively correlated with gender, age, heart rate, alcohol consumption, and family history $(\mathrm{r}=0.069,0.103,0.128,0.062$, and 0.120 , respectively, $\mathrm{P}<$ 0.01 ). No correlations between DBP and weight, height, WC, BMI, TG, TC, HDL-C, LDL-C, FPG, hs-CRP, smoking, or history of diabetes were observed $(\mathrm{P}>0.05)$ (Table 6).

Table 6. Relationship of DBP and cardiovascular risk factors of the patients with PH.
\begin{tabular}{ccccccccc}
\hline & Age & Weight & Height & WC & HR & BMI & TG & TC \\
\hline r & 0.103 & 0.028 & 0.025 & 0.036 & 0.128 & 0.058 & 0.007 & 0.037 \\
Sig. (2-tailed) & 0.001 & 0.357 & 0.418 & 0.236 & 0.000 & 0.057 & 0.816 & 0.218 \\
\hline & HDL-C & LDL-C & FPG & hs-CRP & Smoking & Drinking & & \\
\hline r & 0.016 & 0.031 & 0.010 & 0.014 & 0.027 & 0.062 & & \\
\hline Sig. (2-tailed) & 0.597 & 0.304 & 0.753 & 0.647 & 0.365 & 0.041 & & \\
\hline
\end{tabular}

For abbreviations, see Table 1.

\section{Analysis of PH-associated risk factors by multiple stepwise regressions}

Multiple regression analysis was also used to evaluate the relationship between SBP and DPB with indicators. The regression equation for SBP was SBP $(\mathrm{mmHg})=96.935+$ $(0.554 \mathrm{x}$ gender $)+0.058 \mathrm{x}$ age (years) $\ldots . .+4.348 \mathrm{x}$ family history. The results showed that age, heart rate, TG, and family history all contributed significantly to SBP (Table 7 and Figure 2A).

The regression equation for DBP was DBP $(\mathrm{mmHg})=69.197+(-1.025 \mathrm{x}$ gender $)+0.055$ $\mathrm{x}$ age (years)... $3.102 \mathrm{x}$ family history. The results showed that age, gender, heart rate, BMI, alcohol consumption, and family history all significantly affected DBP (Table 8 and Figure 2B). 
Table 7. Factors affecting SBP from multielement gradual regressive analysis.

\begin{tabular}{lcccrrr}
\hline Dependent variable & Independent variable & Regression coefficient $\beta$ & Standard error & Beta & $\mathrm{t}$ & $\mathrm{P}$ \\
\hline SBP & Constant & 96.935 & 4.113 & - & 23.569 & 0.000 \\
& Age & 0.058 & 0.026 & 0.066 & 2.234 & 0.026 \\
& Heart rate & 0.162 & 0.028 & 0.168 & 5.747 & 0.000 \\
& Triglycerides & 1.163 & 0.271 & 0.147 & 4.287 & 0.000 \\
& Family history & 4.348 & 1.267 & 0.100 & 3.432 & 0.001 \\
\hline
\end{tabular}

A

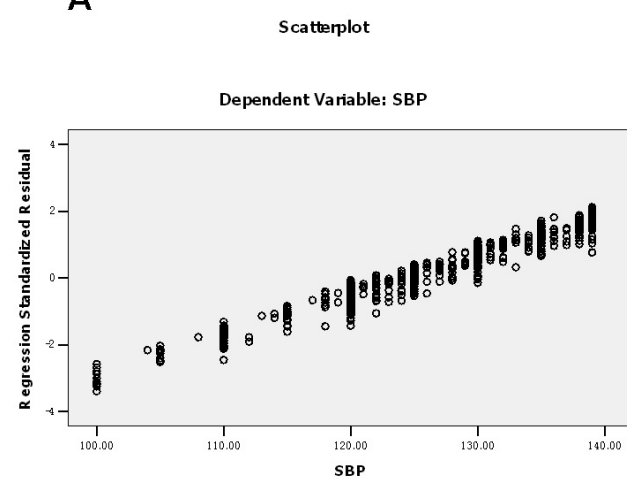

B

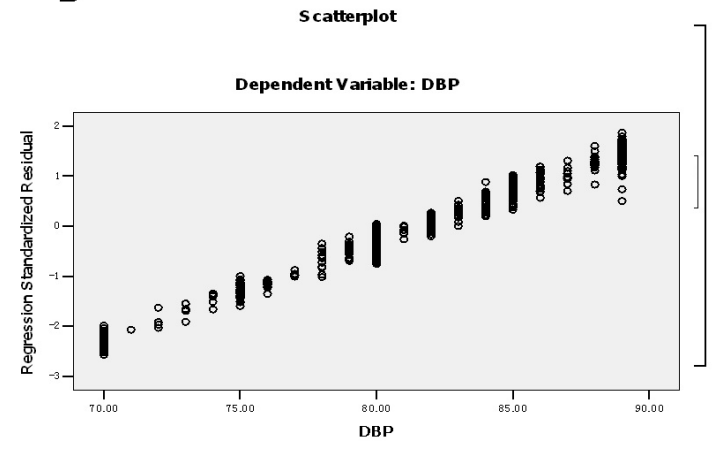

Figure 2. Factors affecting SBP (A) and DBP (B) from multielement gradual regressive analysis.

Table 8. Factors affecting DBP from multielement gradual regressive analysis.
\begin{tabular}{lcccrrr}
\hline Dependent variable & Independent variable & Regression coefficient $\beta$ & Standard error & Beta & \multicolumn{1}{c}{$\mathrm{t}$} & P \\
\hline DBP & Constant & 69.197 & 2.526 & - & 27.391 & 0.000 \\
& Gender & -1.025 & 0.414 & -0.103 & 2.478 & 0.013 \\
& Constant & 0.055 & 0.016 & 0.104 & 3.437 & 0.001 \\
& Age & 0.075 & 0.017 & 0.129 & 4.341 & 0.000 \\
& Heart rate & 0.263 & 0.097 & 0.160 & 2.702 & 0.007 \\
& Triglycerides & -0.951 & 0.443 & -0.070 & 2.148 & 0.032 \\
& Family history & 3.102 & 0.778 & 0.119 & 3.986 & 0.000 \\
\hline
\end{tabular}

\section{Risk factor analysis of $\mathrm{PH}$ population prone to hypertension}

Logistic regression analysis was adopted to determine whether the $\mathrm{PH}$ population might develop hypertension. SBP or DBP after intervention was used as the dependent variable, and SBP, DBP, gender, age, weight, BMI, WC, TG, TC, HDL-C, LDL-C, FPG, hs-CRP, smoking, alcohol consumption, and family history before intervention were independent variables for logistic regression analysis. The results of the model test at each step were statistically significant, showing that TG, hs-CRP, alcohol consumption, and increased SBP were major risk factors for hypertension progression, with values of 1.967, 1.377, 2.197, and 1.995, respectively (Tables 9 and 10).

Logistic regression of DBP indicated that BMI, hs-CRP, alcohol, family history, and increased SBP and DBP were all major risk factors for hypertension, with values of 1.516, $1.847,0.234,2.908,1.749$, and 1.600, respectively (Tables 9 and 11). 
Table 9. Assignments of risk factors of the patient from PH to hypertension.

\begin{tabular}{lccc}
\hline Independent variables & & \multicolumn{2}{c}{ Assignments } \\
\hline Gender & Male $=1$ & Female $=2$ & \\
Age arrange & $20 \sim=1$ & $30 \sim=2$ & $40 \sim=3$ \\
Body weight & $50 \sim=1$ & $70 \sim=2$ & $90 \sim=3$ \\
BMI & $<24=1$ & $>28=3$ & \\
& $24 \sim 28=2$ & & \\
WC & $<80=1$ & $>90=3$ & \\
& $80 \sim 90=2$ & & \\
TG & $<0.4=1$ & $>1.8=3$ & \\
& $0.4 \sim 1.8=2$ & & \\
TC & $<3.0=1$ & $>5.7=3$ & $>2.19=3$ \\
& $3.0 \sim 5.7=2$ & $0.9 \sim 2.19=2$ & \\
HDL-C & $<0.9=1$ & & \\
LDL-C & $<2.0=1$ & $>3.5=3$ & \\
& $2.0 \sim 3.5=2$ & $3.9 \sim 6.19=2$ & \\
FPG & $<3.9=1$ & $0.00 \sim 0.3=2$ & \\
hs-CRP & $<0.00=1$ & $130 \sim 139=2$ & \\
SBP & $120 \sim 129=1$ & & \\
DBP & $80 \sim 84=1$ & & \\
Smoking & $85 \sim 89=2$ & Yes $=2$ & \\
Nrinking & No $=1$ & Yes $=2$ & \\
Family history & No $=1$ & Yes $=2$ & \\
\hline For abreviation & No $=1$ & &
\end{tabular}

For abbreviations, see Table 1.

Table 10. Logistic stepwise regression analysis of risk factors from SBP to hypertension.

\begin{tabular}{|c|c|c|c|c|c|c|c|}
\hline \multirow[t]{2}{*}{ Risk factors } & \multirow[t]{2}{*}{$\beta$} & \multirow[t]{2}{*}{$\mathrm{SE}$} & \multirow[t]{2}{*}{ Wald } & \multirow[t]{2}{*}{ Sig. } & \multirow[t]{2}{*}{ OR } & \multicolumn{2}{|c|}{$95 \% \mathrm{CI}$ for $\operatorname{Exp}(\beta)$} \\
\hline & & & & & & Lower & Upper \\
\hline$\overline{\text { SBP }}$ & 0.691 & 0.219 & 9.910 & 0.002 & 1.995 & 1.298 & 3.067 \\
\hline TG & 0.676 & 0.337 & 4.031 & 0.045 & 1.967 & 1.016 & 3.807 \\
\hline hs-CRP & 0.320 & 0.411 & 0.607 & 0.436 & 1.377 & 0.616 & 3.081 \\
\hline Drinking & 0.787 & 0.373 & 4.452 & 0.035 & 2.197 & 1.058 & 4.565 \\
\hline
\end{tabular}

For abbreviations, see Table 1.

Table 11. Logistic stepwise regression analysis of risk factors from DBP to hypertension.

\begin{tabular}{|c|c|c|c|c|c|c|c|}
\hline \multirow[t]{2}{*}{ Risk factors } & \multirow[t]{2}{*}{$\beta$} & \multirow[t]{2}{*}{ SE } & \multirow[t]{2}{*}{ Wald } & \multirow[t]{2}{*}{ Sig. } & \multirow[t]{2}{*}{ OR } & \multicolumn{2}{|c|}{$95 \%$ CIfor $\operatorname{Exp}(\beta)$} \\
\hline & & & & & & Lower & Upper \\
\hline$\overline{\mathrm{DBP}}$ & 0.470 & 0.234 & 4.037 & 0.045 & 1.600 & 1.012 & 2.532 \\
\hline SBP & 0.559 & 0.246 & 5.179 & 0.023 & 1.749 & 1.081 & 2.832 \\
\hline BMI & 0.416 & 0.243 & 2.927 & 0.087 & 1.516 & 0.941 & 2.440 \\
\hline hs-CRP & 0.614 & 0.390 & 2.473 & 0.116 & 1.847 & 0.860 & 3.969 \\
\hline Drinking & -1.453 & 0.741 & 3.845 & 0.050 & 0.234 & 0.055 & 0.999 \\
\hline Family history & 1.067 & 0.567 & 3.542 & 0.060 & 2.908 & 0.957 & 8.837 \\
\hline
\end{tabular}

For abbreviations, see Table 1.

\section{Compliance results}

The overall compliance was defined as the ratio of total study exposure time to total follow-up time. The compliance of the lifestyle intervention group was $54.5 \%$ (Table 12), and was $52.7 \%$ in the drug group $(\mathrm{P}<0.05)$ (Table 13). 


Table 12. Dependence condition of lifestyle treatment.
\begin{tabular}{lccc} 
& & & Percentage (\%) \\
\hline Dependence on dietary treatment & Values & Cases & 26.44 \\
Dependence on movement treatment & $1 \sim 2$ & 147 & 73.56 \\
& $3 \sim 4$ & 142 & 25.54 \\
74.46 & 414 & 72 \\
\hline
\end{tabular}

Table 13. Incidence of adverse effect.

\begin{tabular}{lcc}
\hline Common side effects & Drug treatment group $(\mathrm{N}=278)$ & Lifestyle + drug treatment $(\mathrm{N}=278)$ \\
\hline Sicchasia & 2 & 3 \\
Distention of head & 6 & 4 \\
Debilitation & 5 & 2 \\
Stuffiness & 3 & 1 \\
Somnolence & 0 & 1 \\
Hypotension & 0 & 0 \\
\hline
\end{tabular}

Compliance with respect to exercise intervention was better than that of dietary intervention, most likely because exercise intervention was easier to adhere to. Nevertheless, self-monitoring and step-by-step goals were still required throughout the follow-up period.

As 1112 individuals were followed-up, we used the Morisky questionnaire to evaluate the compliance of patients with respect to drug intervention. All patients were divided into two groups according to the Morisky score (Table 14). In the drug group, 55.4\% of subjects showed good compliance, whereas the remaining subjects showed poor compliance. Therefore, compliance of lifestyle intervention treatments was better than that of drug intervention.

\begin{tabular}{lcr}
\multicolumn{2}{c}{ Table 14. Dependence condition of administration. } & Percentage (\%) \\
\hline Scores & Cases & 55.4 \\
\hline $0 \sim 1$ & 248 & 44.6 \\
$2 \sim 4$ & 308 & \\
\hline
\end{tabular}

\section{New hypertension, diabetes, and clinical endpoint events}

At the end of the follow-up period, there were 67 new cases of hypertension, 32 new cases of diabetes, and 12 new cases of endpoint events. Compared with the control group, the number of new hypertensive and diabetes cases were substantially decreased in the three intervention groups $(\mathrm{P}<0.05)$. New diabetes cases were lower in the lifestyle + drug group compared with the lifestyle and drug intervention groups $(\mathrm{P}<0.05)$. Overall, the number of cardiovascular endpoint events did not differ significantly among the three groups $(\mathrm{P}>0.05)$ (Table 15).

Table 15. Termination events (cases).
\begin{tabular}{lcccc} 
& & & & \\
\hline Items & Control & Lifestyle & Drug & Lifestyle + drug \\
\hline New onset hypertension & 34 & $10^{*}$ & $14^{*}$ & 9 \\
New onset diabetes cardiovascular & 10 & 7 & 3 & $6^{*} \Delta$ \\
Termination events & 4 & 3 & 3 & 2 \\
\hline
\end{tabular}


In the final stage of observations, 34 cases had advanced to hypertension, 10 cases progressed to diabetes, and four cardiovascular events were reported in the control group. Compared with the three intervention groups, the incidence of hypertension, diabetes, and cardiovascular events were all significantly higher in the control group, suggesting that these three types of intervention were effective, feasible, and worthy of promotion to the general population.

\section{DISCUSSION}

The incidence of $\mathrm{PH}$ is high in both developed and developing countries. Hypertension risk reaches $90 \%$ in the 55 -year-old or older normal blood pressure population. The average level of cardiovascular risk factors is significantly higher in the $\mathrm{PH}$ population compared to the normal blood pressure population (Dong et al., 2006). In addition, the PH population has been associated with an increased tendency for target organ damage, such as heart and kidney damage (Kim et al., 2007; Mineeva et al., 2008). Based on the high incidence, risk factors of hypertension, and early heart and kidney damage of the $\mathrm{PH}$ population, we applied different intervention methods to $\mathrm{PH}$ patients with cardiovascular disease risk in this study. We observed a significant reduction in new cases of hypertension, diabetes, and cardiovascular endpoint events in the intervention groups compared to the control group, which provides a basis for the primary and early prevention of hypertension (Sun et al., 2005, 2007; Lee et al., 2009).

As PH is itself a cardiovascular disease risk factor, its risk factors are similar to those of cardiovascular disease, including age, gender, family history of hypertension, smoking, alcohol consumption, increased weight and obesity, high blood lipid levels, FPG, insulin resistance, hs-CRP, high uric acid, micro-albuminuria, arterial compliance, carotid intima-media thickness, early plaque formation, and organ damage. In our study, we determined the effect of several indicators on SBP and DBP. Results of partial correlation analyses showed that SBP was positively correlated with age, heart rate, TG, FPG, and family history. DBP was positively correlated with gender, age, heart rate, alcohol consumption, and family history. No correlations were found between other indicators and SBP or DBP. We also performed stepwise multiple regression analysis using SBP and DBP as the dependent variables and other indicators as the independent variables. Results showed that age, heart rate, TG, and family history were the main influencing factors of SBP. Similarly, age, gender, heart rate, BMI, alcohol consumption, and family history were the main influential factors of DBP. Intervention for major risk factors affecting the population, active prevention, control of blood pressure, and prevention of $\mathrm{PH}$ and its associated risk factors have been shown to be beneficial in preventing cardiovascular disease (Lee et al., 2006; Erdogan et al., 2007; Mineeva et al., 2008).

On a global scale, individuals with PH are more likely to develop hypertension and have a higher risk of cardiovascular and cerebrovascular diseases compared to individuals with normal blood pressure. The incidence of hypertension is approximately $19.3 \%$ if $\mathrm{PH}$ is present as of childhood (Liang, 2011). Within the PH population that developed hypertension, 20.77, 37.03 , and $42.20 \%$ showed isolated systolic hypertension, isolated diastolic hypertension, and both systolic and diastolic hypertension, respectively. Factors such as age, gender, WC, and TC show different contributions on the progression of $\mathrm{PH}$ into various types of hypertension, and cardiovascular risk factors show different effects in different age groups (Franklin et al., 2001; McEniery et al., 2010; Huang et al., 2011). Elderly patients mainly manifest arterial elasticity decline, whereas young patients mainly manifest increases in small arterial resistance. These obser- 
vations suggest that different preventive measures should be adopted in $\mathrm{PH}$ patients of different age groups. In the present study, we found that TG, hs-CRP, alcohol consumption, and increase in SBP were all major risk factors in the progression of $\mathrm{PH}$ to systolic hypertension, whereas BMI, hs-CRP, alcohol consumption, family history, and increased SBP and DBP were the main risk factors for the progression of $\mathrm{PH}$ to hypertension. These results suggested that reducing TG, drinking, and the SBP level could prevent SBP progression to hypertension. Controlling the diet to avoid obesity, and controlling temperament to reduce SBP and DBP can prevent DBP from progressing to hypertension. Additionally, people with a family history of hypertension should monitor their blood pressure on a regular basis, and adopt early intervention to prevent the progression of DBP to hypertension. The above-mentioned risk factors have all contributed to the continuous increase in cases of adulthood hypertension, which results in serious complications caused by unstable blood pressure. Therefore, it is necessary to take serious steps toward controlling hypertension at an early stage, even before its onset.

hs-CRP is a non-specific, but sensitive, biological indicator of inflammation. As an alternative indicator of the cytokine activation degree, hs-CRP is a partial predictor of the development and prognosis of cardiovascular disease. Serum hs-CRP levels are significantly increased in patients with $\mathrm{PH}$ (Wu et al., 2008), which is a risk factor of high blood pressure and cardiovascular and cerebrovascular disease. Bautista et al. (2001) demonstrated that serum hs-CRP concentration was an independent risk factor for primary hypertension. The results of the present study also showed that hs-CRP was a major risk factor in the progression of $\mathrm{PH}$ to hypertension. There have been no similar studies. hs-CRP can therefore be used as a marker for the early detection of PH indicators, and provides a theoretical basis for very early diagnoses of PH. Levels of hs-CRP were not significantly different between the control group and other groups before intervention $(\mathrm{P}>0.05)$. After intervention, hs-CRP was significantly different between the control group and both the lifestyle intervention group and the lifestyle + drug intervention group $(\mathrm{P}<0.05)$, but did not differ with respect to the drug group $(\mathrm{P}>0.05)$. These observations suggested that lifestyle interventions were effective in controlling blood pressure and reduced hs-CRP. High-sensitivity CRP could serve as an indicator for the turnover of blood pressure, which has little significance for drug monitoring in blood pressure control. It is necessary to expand the scale of clinical trials in order to adopt appropriate interventions in the treatment of inflammatory mechanisms, to understand the development and outcome of intervention after $\mathrm{PH}$, and to better monitor the occurrence and development of hypertension. Therefore, for effective control of hypertension and other cardiovascular and cerebrovascular diseases, more attention should be paid to the basis and clinical intervention of hs-CRP during the PH stage (Timmermans et al., 1993; Julius et al., 2006; Márquez-Celedonio et al., 2009).

The JNC7 recommended improving lifestyle as the main prevention of cardiovascular disease in PH patients. However, for patients with renal disease and diabetes, lifestyle improvements might not effectively decrease blood pressure to $130 / 80 \mathrm{mmHg}$, and drug therapy should be considered (Kim et al., 2007). The European hypertension guidelines of 2007 (Mancia et al., 2007) strongly recommended that people with PH who suffer from cardiovascular complications should adopt drug therapy in order to reduce their risk of cardiovascular events.

In the present study, besides lifestyle intervention, we also treated PH patients with risk factors with antihypertension drugs and basic drugs. The results showed that the combination of lifestyle intervention and drugs showed better effects on blood pressure control compared to either lifestyle intervention or basic drug treatment independently $(\mathrm{P}<0.05)$. There were obvious differences in blood pressure control with respect to SBP and DBP among the 
four groups after intervention. Bonferroni's pairwise comparison analysis showed that SBP and DBP were significantly different between the control group and the three intervention groups $(\mathrm{P}<0.05)$, and that $\mathrm{DBP}$ was significantly different between the lifestyle + drug intervention group and the other groups $(\mathrm{P}<0.05)$. However, DBP was not significantly different between the lifestyle and drug treatment groups $(\mathrm{P}>0.05)$, suggesting that simple lifestyle or drug intervention on their own have no obvious effects on DBP control.

There is also a possibility that due to the small sample size, the simple drug treatment might not have reached its maximum effect during the study. Therefore, this area should be investigated further in larger samples. The decline of SBP and DBP was significantly different between the control group and the three intervention groups. Furthermore, the reduction in SBP and DBP was greater in the lifestyle + drug intervention group compared to the other three groups $(\mathrm{P}<0.05)$, which suggested that the combination of lifestyle and medication intervention has a better effect in controlling SBP and DBP. Compound Hypertension Tablets is a complex reagent, and its mechanisms of action are rather complicated. The drug has diuretic effects through the inhibition of renal cortical dilution segments, from re-absorbing sodium, and increasing the discharge of urinary sodium and urinary chlorine. In the early stage of the treatment, extracellular fluid volume is reduced, and blood volume and cardiac output are decreased, thus lowering the blood pressure. In the later stage of treatment, total peripheral resistance is reduced, which plays a major role in reducing the blood pressure. The selection criteria of antihypertensive drugs include good antihypertensive effect, obvious control of symptoms, reduced side effects, and good dependence for long-term use. Compound Hypotensive Tablets essentially meet the above-mentioned criteria, and it is safe, effective, and suitable for clinical applications. At the end of the follow-up period of the present study, the average weight, BMI, and WC were all lower in the intervention groups after intervention. In the control group, average weight, BMI, and WC all increased and were drastically different from those of the intervention groups. In the lifestyle intervention group and the lifestyle + drug intervention group, weight, BMI, and WC were all reduced after intervention, suggesting that lifestyle intervention could effectively control obesity. After intervention, the TG and FPG levels differed significantly between the control group and both the lifestyle and the lifestyle + drug intervention groups $(\mathrm{P}<0.05)$, whereas the difference was not significant between the control and the drug groups $(\mathrm{P}>0.05)$, suggesting that lifestyle intervention could decrease TG and FPG and that medication intervention had a minor effect in controlling these factors. At the end of the follow-up period, there were 67 new cases of hypertension, 32 new cases of diabetes, and 12 cases of endpoint events. Compared with the control group, the number of new hypertension and diabetes cases were reduced in the lifestyle, drug, and lifestyle + drug intervention groups. Compared with the lifestyle and drug intervention groups, the number of new diabetes cases was reduced in the lifestyle + drug intervention group. The number of cardiovascular endpoint events did not differ significantly among the three groups. In conclusion, results of our study suggest that the progression of PH to hypertension is inevitable, and measures can only be taken to slow down its development, but cannot prevent it.

\section{Conflicts of interest}

The authors declare no conflict of interest. 


\section{REFERENCES}

Bautista LE, Lopez-Jaramillo P, Vera LM, Casas JP, et al. (2001). Is C-reactive protein an independent risk factor for essential hypertension? J. Hypertens. 19: 857-861.

Chobanian AV, Bakris GL, Black HR, Cushman WC, et al. (2003). The seventh report of the joint national committee on prevention, detection, evaluation and treatment of high blood pressure: the JNC7 report. JAMA 289: 2560-2572.

Chockalingam A, Ganesan N, Venkatesan S and Gnanavelu G (2005). Pattems and predictors of prehypertension among "healthy" urban adults in India. Angiology 56: 557-563.

Choi KM, Park HS, Han JH, Lee JS, et al. (2006). Prevalence of prehypertension and hypertension in a Korean population: Korean national health and nutrition survey 2001. J. Hypertens. 24: 1515-1521.

Dong Z, Cuifen L and Wei W (2006). High normal blood pressure population 10 years of cardiovascular disease risk analysis. Chin. J. heart Cerebrovasc. Dis. 8: 733-736.

Erdogan D, Yildirim I, Ciftci O, Ozer I, et al. (2007). Effects of normal blood pressure, prehypertension, and hypertension on coronary microvascular function. Circulation 115: 593-599.

Fang JN, Xiong YH and Jin ZG (2010). Yanbian state rural residents of high normal blood pressure and its influencing factors. Chin. Publ. Health 26: 257-259.

Franklin SS, Jacobs MJ, Wong ND, L'Italien GJ, et al. (2001). Predominance of isolated systolic hypertension among middle-aged and elderly US hypertensives: Analysis based on national health and nutrition examination survey (NHANES) III. J. Hypertens. 37: 869-874.

Grotto I, Grossman E, Huerta M and Sharabi Y (2006). Prevalence of prehypertension and associated cardiovascular risk profiles among young Israeli adults. Hypertension 48: 254-259.

Huang Z, Wu S and Gao J (2011). Of prehypertension population blood pressure and its affecting factors. Chin. J. Hypertens. 19: 630-635.

Julius S, Nesbitt SD, Egan BM, Weber MA, et al. (2006). Feasibility of treating prehypertension with an angiotensinreceptor blocker. N. Engl. J. Med. 354: 1685-1697.

Kim BJ, Lee HJ, Sung KC, Kim BS, et al. (2007). Comparison of microalbuminuria in 2 blood pressure categories of prehypertensive subjects. Circ. J. 71: 1283-1287.

Lee JE, Kim YG, Choi YH, Huh W, et al. (2006). Serum uric acid is associated with microalbuminuria in prehypertension. Hypertension 47: 962-967.

Lee YH, Kweon SS, Choi JS, Rhee JA, et al. (2009). Association of blood pressure levels with carotid intima-media thickness and plaques. J. Prev. Med. Public Health 42: 298-304.

Liang Y (2011). Childhood Hypertension Forecast the Prospective Study of Adult Hypertension and Target Organ Damage [D] Beijing. Chinese Peking Union Medical College, Sun Gang.

Liu M, Chen XY and Liu R (2010). Crowd of blood pressure, high normal uric acid status and related factors. Sichuan Med. 31: 704-705.

Mancia G, de Backer G, Dominiczak A, Cifkova R, et al. (2007). Guidelines for the management of arterial hypertension: The task force for the management of arterial hypertension of the European society of hypertension (ESH) and of the European society of cardiology (ESC). Eur. Heart J. 28: 1462-1536.

Márquez-Celedonio FG, Texon-Fernandez O, Chavez-Negrete A, Hernandez-Lopez S, et al. (2009). Clinical effect of lifestyle modification on cardiovascular risk in prehypertensives: PREHIPER I study. Rev. Esp. Cardiol. 62: 86-90.

McEniery CM, Kaisa M and Maki-Petaja KM (2010). The impact of cardiovascular risk factors on aortic stiffness and wave reflections depends on age: the Anglo-Cardiff Collaborative Trial (ACCT III). J. Hypertens. 56: 591-597.

Mineeva EE, Gvozdenko TA and Antoniuk MV (2008). Diastolic dysfunction-predictor of cardiac remodeling in arterial hypertension in young males. Klin. Med. 86: 23-25.

Sun G, Zhang X and Ding YC (2005). Changes in blood pressure above normal values of arterial compliance Chin. J. Hypertens. 13: 273-276.

Sun J and Zhao D (2005). Blood pressure level of the Beijing 2740 people in the region 10 years (1922-2002) changes. $J$. Hypertens. 13: 115-119.

Sun R, Gao YY and Xian YX (2010). Shandong coastal areas of young prehypertensive popular features. Chin. J. Hypertens. 18: 486-490.

Sun Z, Zheng L, Wei Y, Li J, et al. (2007). Prevalence and risk factors of the rural adult people prehypertension status in Liaoning province of China. Circ. J. 71: 550-553.

Timmermans PB, Wong PC, Chiu AT, Herblin WF, et al. (1993). Angiotensin II receptors and angiotensin II receptor antagonists. Pharmacol. Rev. 45: 205-251.

Tsai PS, Ke TL, Huang CJ, Tsai JC, et al. (2005). Prevalence and determinants of prehypertension status in the Taiwanese general population. J. Hypertens. 23: 1355-1360. 
Vasan RS, Larson MG, Leip EP, Evans JC, et al. (2001). Impact of high-normal blood pressure on the risk of cardiovascular disease. N. Engl. J. Med. 345: 1291-1297.

Wang C, Liuyong J and Liu WK (2011). Beijing 1315 policemen blood pressure level epidemiological analysis. Chron. Dis. Prev. Contr. 19: 12-14.

Wang Y and Wang QJ (2004). The prevalence of prehypertension and hypertension among US adults according to the New Joint National Committee Guidelines: new challenges of the old problem. Arch. Intern. Med. 164: 2126-2134.

Wu SJ, Liang J and Zhang J (2008). Of prehypertension crowd serum C reaction protein level. Mod. Lab. Med. 23: 95-96.

Zhao D, Cuifen L and Wei W (2006). High normal blood pressure, the crowd of 10-year incidence of cardiovascular disease risk analysis. Chin. J. Heart Cerebrovasc. Dis. 8: 730-733. 